\title{
CW Leonis, IRC+10216
}

\author{
G. R. Knapp \\ Department of Astrophysical Sciences, Princeton University, Princeton, \\ NJ 08544, USA
}

\begin{abstract}
CW Leo is the nearest and brightest example of a highly evolved carbon Mira variable red giant, and is surrounded by an extensive dusty, molecular circumstellar shell produced by mass loss. Since the shell heavily obscures the star, most of the information about CW Leo comes from observations of its shell, whose radial properties encode a history of the star as well as of the interaction between the outflowing gas and the interstellar radiation field. The shell of CW Leo contains a rich variety of molecules, from salts to cyanide, whose isotopic composition shows the effect of nuclear processing in the stellar interior and give information about the formation and composition of the circumstellar dust. This nearby star allows us a detailed look at one of the most eventful and interesting phases in a star's evolution.
\end{abstract}

\section{Introduction}

The variable star CW Leo, or, to use its discovery name, IRC+10216 (Neugebauer \& Leighton 1969) is a nearby, highly evolved, high luminosity, long-periodvariable carbon star, a type of chemically peculiar star characterized by photospheric abundances $n(\mathrm{C})>n(\mathrm{O})$ and the presence of s-process elements. This star is losing mass at a high rate, several $\times 10^{-5} \mathrm{M}_{\odot} \mathrm{yr}^{-1}$, and has been doing so for at least several tens of thousands of years (e.g. Young, Phillips, \& Knapp 1993). Its importance to astronomy lies in its proximity to the Sun; the large angular size of its envelope enables detailed studies of the mass loss process, the late stages of stellar evolution, and the evolution of the composition of the interstellar medium. CW Leo is thus a paradigm object for studies of mass losing giant stars, and is (probably) not exotic at all: rather, it is an example of the exotic physical and chemical processes that take place at the late stages of stellar evolution (Olofsson 1999).

This review attempts a summary of the observational properties of CW Leo, beginning in the next chapters with a short discussion of the object's history and vital statistics. Subsequent chapters discuss the chemistry and physical structure of the circumstellar envelope. In particular, new high resolution observations of $\mathrm{CW}$ Leo reveal a wealth of information on the surprisingly complex and detailed structure in the inner envelope. 


\section{CW Leo: A Short History}

IRC+10216 was discovered in 1967 by the Two Micron Sky Survey conducted by Neugebauer \& Leighton (1969). It is detected on the red plate, but not the blue, of the Palomar Observatory Sky Survey at a red magnitude of about 18. The optical image is slightly elliptical, with an angular size of about $2^{\prime \prime} \times 1^{\prime \prime}$ (Becklin et al. 1969). The enormous R-K color already indicates the extreme nature of this object. Herbig \& Zappala (1970) obtained a spectrum showing the presence of carbon features, and concluded that the object is a very highly reddened cool N type carbon star at a distance of $290 \mathrm{pc}$. However, since CW Leo is so heavily obscured, little else is known from its optical spectrum: the abundances of s-process elements have not been investigated, for example.

Shortly after its discovery, Solomon et al. (1971) found strong $2.6 \mathrm{~mm}$ $\mathrm{CO}(1-0)$ rotational line emission from IRC +10216 , but with a line profile of quite different shape from the profiles seen for molecular clouds. Molecular cloud emission line profiles are roughly Gaussian in shape, with widths of a few $\mathrm{km} / \mathrm{sec}$. The line profile of IRC+10216 is much broader, about $30 \mathrm{~km} / \mathrm{sec}$, and has a roughly parabolic shape. Further, the line-emitting region is small, only a few arcminutes (a fraction of $1 \mathrm{pc}$ ), while nearby molecular clouds typically have emission over many degrees, corresponding to cloud sizes of many pc. These features are explained by Morris' (1975) line emission model, in which the line profile results from a roughly spherical envelope expanding at a constant velocity of $\sim 14 \mathrm{~km} / \mathrm{sec}$ and formed from a steady mass loss rate of a few $\times 10^{-5} \mathrm{M}_{\odot} \mathrm{yr}^{-1}$. This model demonstrates the power of molecular line observations of the circumstellar envelopes of evolved stars to measure fundamental quantities: the systemic velocity (the mean velocity of the molecular line), the terminal outflow velocity (the molecular line width), and the mass loss rate (the line brightness), and has been used as the standard model in essentially all subsequent analyses of line emission from circumstellar molecular envelopes. These models have by now been applied to observations of several hundred envelopes (e.g. Habing 1996; Olofsson et al. 1996). The envelopes show a wealth of chemical species, particularly that of IRC+10216, and the extent of the envelope provides a time map of various processes: the mass loss history, the chemical evolution of the star, and further chemical processes in the outflowing envelope, which provide insight into the effect of the interstellar radiation field on molecular chemistry (e.g. Glassgold 1996). The circumstellar chemistry of IRC+10216 will be discussed in Section 4 .

The broad-band spectral energy distribution (SED) of IRC+10216 is shown in Fig. 1, constructed from observations of emission at optical to radio wavelengths. Essentially all of the radiation from this object is emitted at wavelengths longer than $5 \mu \mathrm{m}$. The SED peaks at about $11 \mu \mathrm{m}\left(\mathrm{S}_{\nu}\right)$, and IRAS observations show that IRC +10216 is the third brightest $12 \mu \mathrm{m}$ source in the sky, after the Galactic Center and the massive-star forming complex containing $\eta$ Carinae. The circumstellar extinction is extremely high even at $2 \mu \mathrm{m}$ (cf. also Fig. 2), and the scatter in the data points at a given wavelength is due to the variability of the object, leading to its variable star designation, CW Leo, in 1976. The spectral energy distribution shows no sign of the silicate emission feature at $9.7 \mu \mathrm{m}$, and the broad-band SED can be well reproduced by re-emission of the starlight by amorphous carbon dust. 


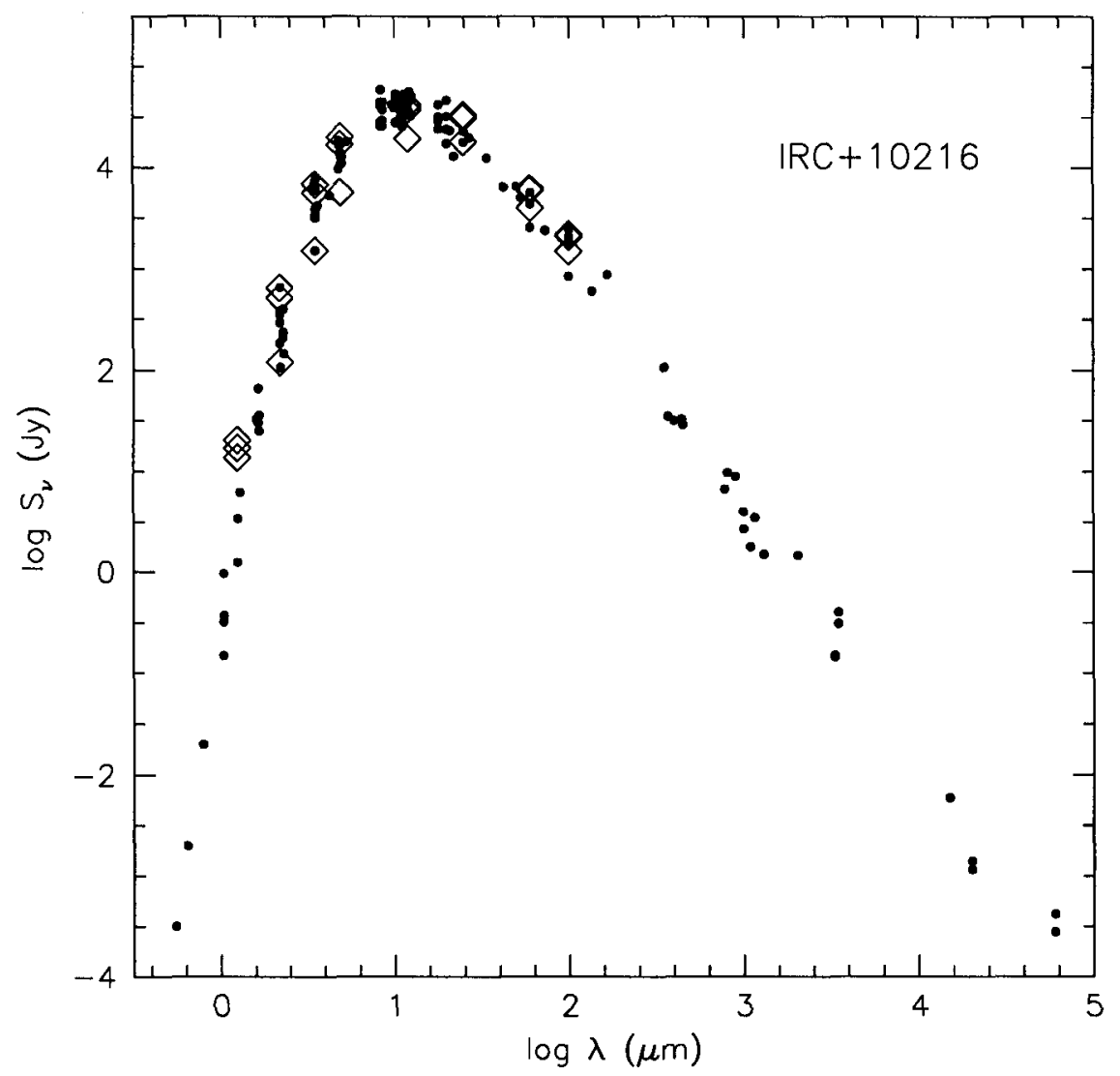

Figure 1. Broad-band spectral energy distribution of IRC+10216. The infrared data are taken from the compilation of Gezari et al. (1993), and the submillimeter and radio data from Sandell (1994) and Knapp et al. (1994). The diamonds are the global flux densities measured by the COBE satellite at three epochs. 


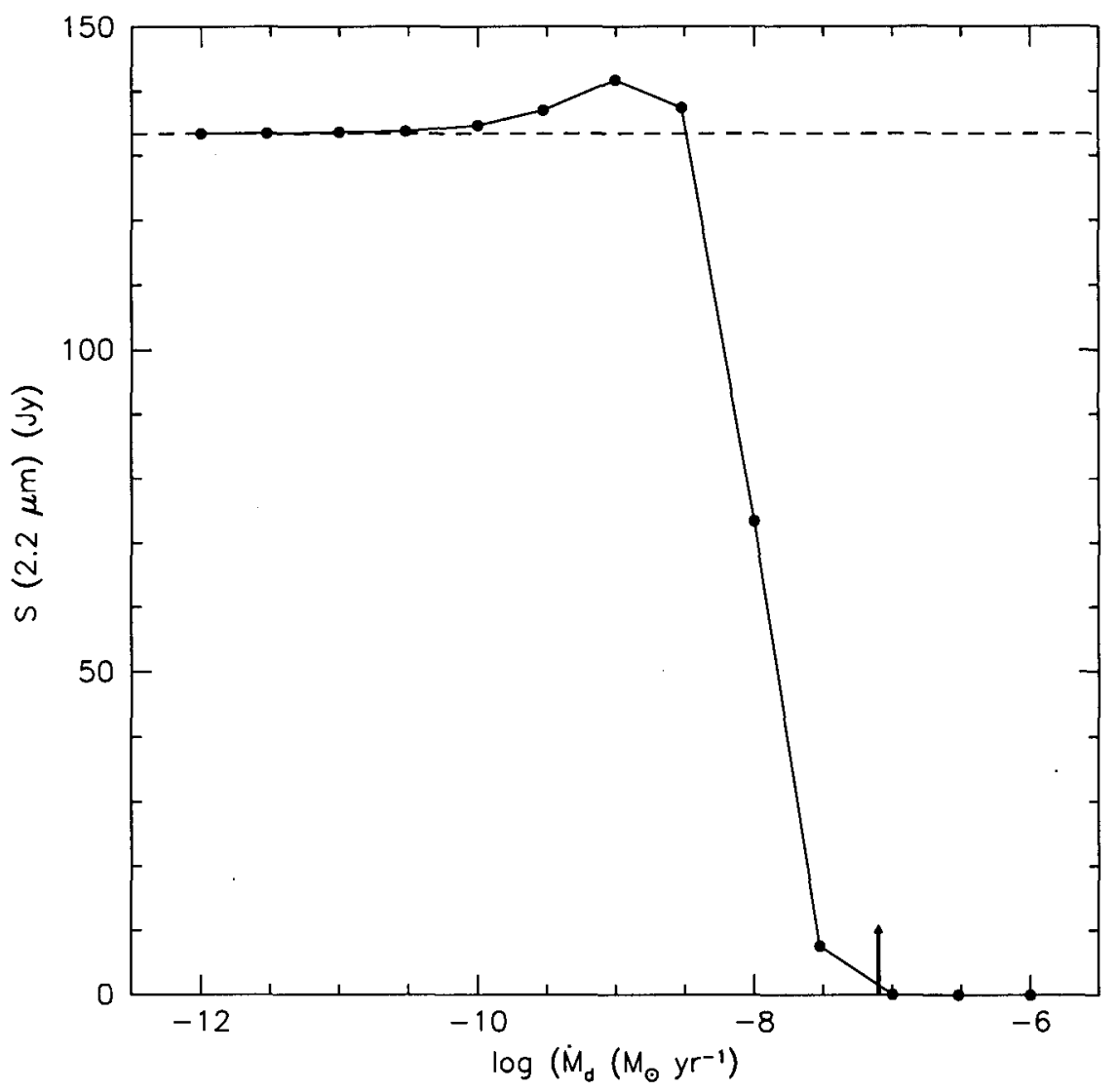

Figure 2. Emergent $2 \mu \mathrm{m}$ flux density for an IRC+10216-like star $\left(L=10^{4} \mathrm{~L}_{\odot}, T_{\text {eff }}=2500 \mathrm{~K}\right)$ at a distance of $1 \mathrm{kpc}$, versus dust ejection rate in $\mathrm{M}_{\odot} \mathrm{yr}^{-1}$. The dashed line shows the photospheric flux density, and the heavy arrow indicates the approximate dust ejection rate for IRC +10216 . The model assumes amorphous dust grains condensing at $1000 \mathrm{~K}$ above the photosphere, an outflow velocity of $14 \mathrm{~km} / \mathrm{sec}$, and a gas-to-dust ratio of 300 by mass. 
Fig. 2 illustrates the dependence of the SED on mass loss rate. For optically thin envelopes, the $2 \mu \mathrm{m}$ flux density at first increases slightly over the photospheric value with increasing mass loss rate, but then declines very rapidly as the envelope opacity increases. Thus carbon stars with mass loss rates in excess of a few $\times 10^{-6} \mathrm{M}_{\odot} \mathrm{yr}^{-1}$, like IRC+10216, are essentially undetectable at optical to mid infrared wavelengths, and must be found across the galaxy by mid-IR sky surveys like IRAS.

\section{Vital Statistics}

CW Leo is at position $\alpha=09^{\mathrm{h}} 47^{\mathrm{m}} 57^{\mathrm{s}} \cdot 4, \delta=+13^{\circ} 16^{\prime} 43^{\prime \prime} \cdot 7(2000), l=$ $221^{\circ} 45, b=+45^{\circ} 06$. Its radial velocity is $-26 \mathrm{~km} / \mathrm{sec}$, and the wind outflow velocity is $14.5 \mathrm{~km} / \mathrm{sec}$. Its spectral type is C9.5 (Herbig \& Zappala 1970). Le Bertre (1982) showed that it is a Mira variable, with a period of $649 \mathrm{~d}$ and an amplitude (at $\mathrm{K}$ ) of about 0.5 magnitudes. At this time, there is no accurate measurement of the distance. Its bolometric flux (cf. Fig. 1) suggests a distance of $100-150 \mathrm{pc}$ if its luminosity is typical of luminosities on the asymptotic giant branch (AGB) - see the detailed discussion by Groenewegen et al. (1998). In principle, a distance estimate could be obtained from the $2 \mu \mathrm{m}$ magnitude or from the period $-K$ luminosity relationship, but the PL relationship for AGB stars is not well established (see for example Groenewegen \& Whitelock 1996), and more to the point, the $2 \mu \mathrm{m}$ optical depth of the circumstellar dust shell is large (see Figs. 1 and 2) so that correction for it will be very uncertain. Further, as discussed below, it is likely that most of the $2 \mu \mathrm{m}$ flux is due to scattered light.

At a distance of $300 \mathrm{pc}$ or less, the parallax should be measurable, at least roughly. However, $\mathrm{CW}$ Leo is far too faint at optical wavelengths to be included in the Hipparcos or ground-based astrometric missions, while at infrared wavelengths (cf. Dahn et al. 2002) the measured errors are larger than expected (H. Harris, private communication), frustrating attempts to measure the parallax. This is almost certainly due to variable structure in the inner envelope, as discussed below. It is likely that a reliable parallax for CW Leo will remain elusive until astrometry with millimeter-wave and short-wavelength radio arrays (for example the Atacama Large Millimeter Array or the Ultra Sensitive Array) become possible. At these wavelengths the dust is transparent enough that most of the emission comes directly from the photosphere. In the following, a (very uncertain) distance of $120 \mathrm{pc}$ (cf. the discussion by Groenewegen et al. 1998, for example) will be assumed where necessary.

For similar reasons, the angular diameter of CW Leo has also proved to be hard to measure. Early speckle measurements by Foy et al. (1979), for example, find an angular diameter of 450 mas at $2 \mu \mathrm{m}$, corresponding to a radius of $4 \times 10^{14} \mathrm{~cm}$ and an effective temperature of $760 \mathrm{~K}$ - thus the observations are almost certainly of scattered light from blobs of circumstellar material rather than of the star itself. More recent speckle observations (Weigelt et al. 1999) separate several contributions to the $2 \mu \mathrm{m}$ emission; that identified with the star itself has a diameter of $\sim 4 \mathrm{AU}$, giving an effective temperature of about $2000 \mathrm{~K}$ (see also Haniff \& Buscher 1998; Chandrasekhar \& Mondal 2001). 
The SED shown in Fig. 1 can be reproduced by a dust ejection rate of $6 \times 10^{-8} \mathrm{M}_{\odot} \mathrm{yr}^{-1}$, while molecular line observations give a gas loss rate of about $2 \times 10^{-5} \mathrm{M}_{\odot} \mathrm{yr}^{-1}$ (Jura 1983, 1994; Sahai 1987; Huggins et al. 1988; Truong Bach et al. 1991; Keady \& Ridgway 1993; Olofsson et al. 1996; Crosas \& Menten 1997; Skinner et al. 1998; Winters et al. 2000; Schöier \& Olofsson 2001), with suggestions in several papers of time variability in the mass loss rate. The gas to dust ratio in the envelope is thus about 400 by mass.

How large is the circumstellar envelope? Maps of the CO rotational lines (Huggins et al. 1988; K. Young \& G. Knapp, unpublished) give an outer radius of about $170^{\prime \prime}-200^{\prime \prime}$ (see Fig. 3). However, as the gas flows into the interstellar medium, the molecules are dissociated by the interstellar radiation field (Mamon, Glassgold, \& Huggins 1988), which determines the extent of the CO envelope. The $\mathrm{CO}$ observations give lower limits to the envelope radius $(0.1 \mathrm{pc})$, the envelope age $\left(7000\right.$ years) and mass $\left(0.2 \mathrm{M}_{\odot}\right)$. The envelope extent as measured in scattered light is of similar size (Tamura et al. 1988) and there are some suggestions in the IRAS $100 \mu \mathrm{m}$ maps, which measure the emission from circumstellar dust, that the envelope is larger (4!6 \pm 0.3 , Young et al. 1993). However, this size cannot be measured with any certainty because of the very bright central source. Finally, observations and models of the molecular envelope suggest that the $\mathrm{C} / \mathrm{O}$ ratio in the envelope is $\sim 1.4$ (Groenewegen 1997; Groenewegen et al. 1998; Winters et al. 2000).

CW Leo is thus at an advanced stage of evolution on the AGB, as shown by its long period, large amplitude, high mass loss rate, and large $\mathrm{C} / \mathrm{O}$ ratio. The relatively large distance from the galactic plane, and the radial velocity, suggest that the star is a member of the thick disk population, while the mass of the circumstellar envelope suggests a progenitor mass a bit larger than $1 \mathrm{M}_{\odot}$. It will be assumed in the following that CW Leo is a typical highly evolved carbon star with progenitor mass about $1 \mathrm{M}_{\odot}$.

\section{Envelope Chemistry}

CW Leo is cool enough that its envelope is expected to be completely molecular, and the molecular abundances of the circumstellar gas are determined by the photospheric chemistry. In contrast to the chemistry of the interstellar medium (high temperature and density, negligible ionization fraction, versus low temperature and density, molecular abundances largely determined by ionmolecule chemistry) that of circumstellar envelopes does not produce molecular ions $\left(\mathrm{HCO}^{+}\right.$, for example) and does produce large abundances of saturated species $\left(\mathrm{CH}_{4}, \mathrm{SiH}_{4}\right.$, etc.) However, the expansion of the circumstellar cloud into the interstellar medium at approximately constant mass loss rate and outflow speed sets up a density gradient $\left(\mathrm{r}^{-2}\right)$, and molecules are photodissociated by interstellar UV. To first order, this occurs at a distance from the star where the flux of outflowing molecules is equal to that of the interstellar dissociating field, so that abundant molecules like $\mathrm{CO}$ survive to much larger radii than do molecules with low abundances. This situation differs from that in molecular clouds, which are much larger and of approximately constant density, and for which the transition between the atomic and molecular phases occurs for all species in approximately the same thin region at the edge of the cloud, where 


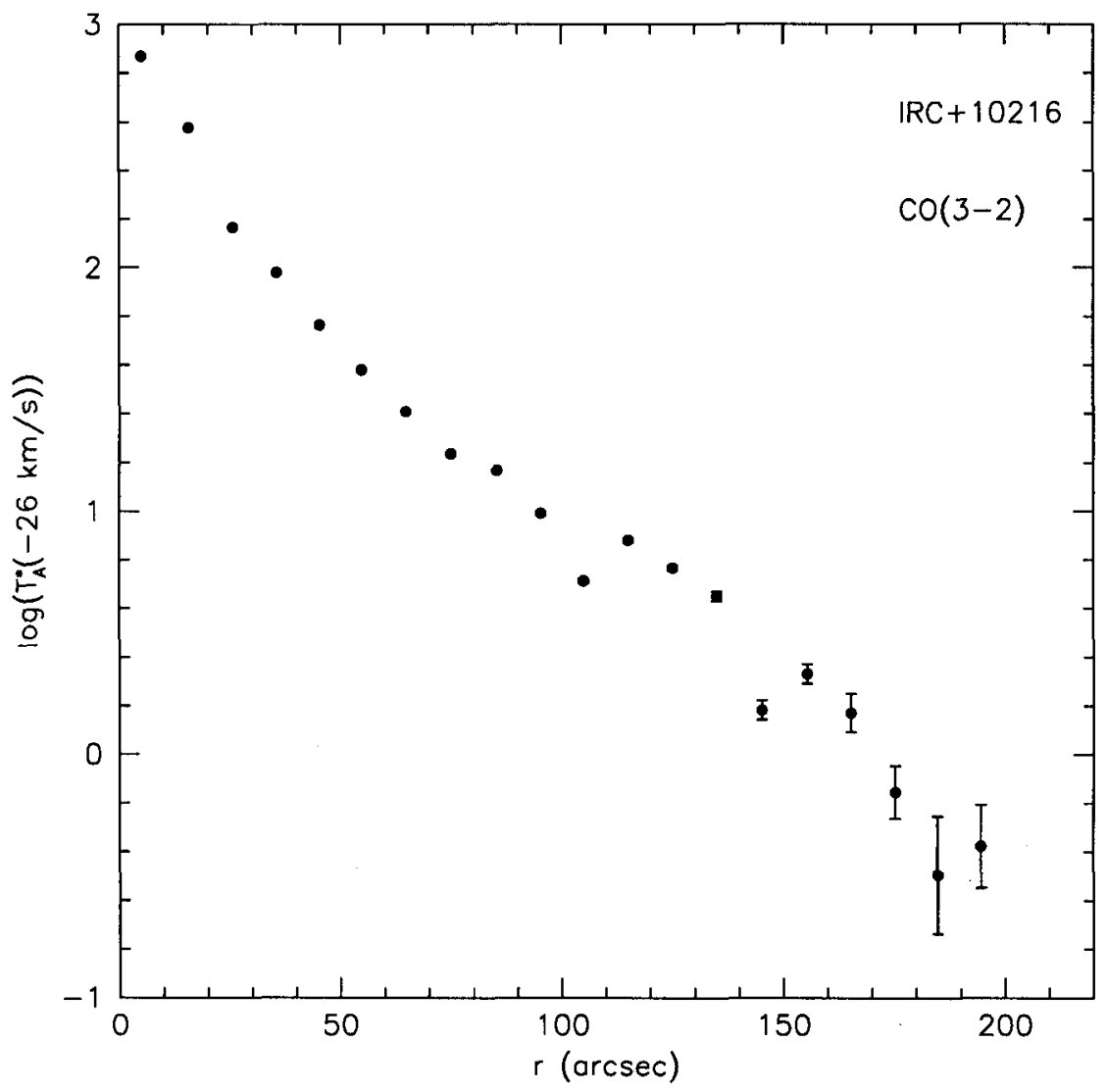

Figure 3. Azimuthally averaged $\mathrm{CO}(3-2)$ line brightness at $V_{\mathrm{LSR}}=-26 \mathrm{~km} / \mathrm{sec}$ (the stellar systemic velocity) versus radius, observed with the Caltech Submillimeter Observatory's 10.4-m telescope. The telescope half-power beamwidth is $20^{\prime \prime}$. 


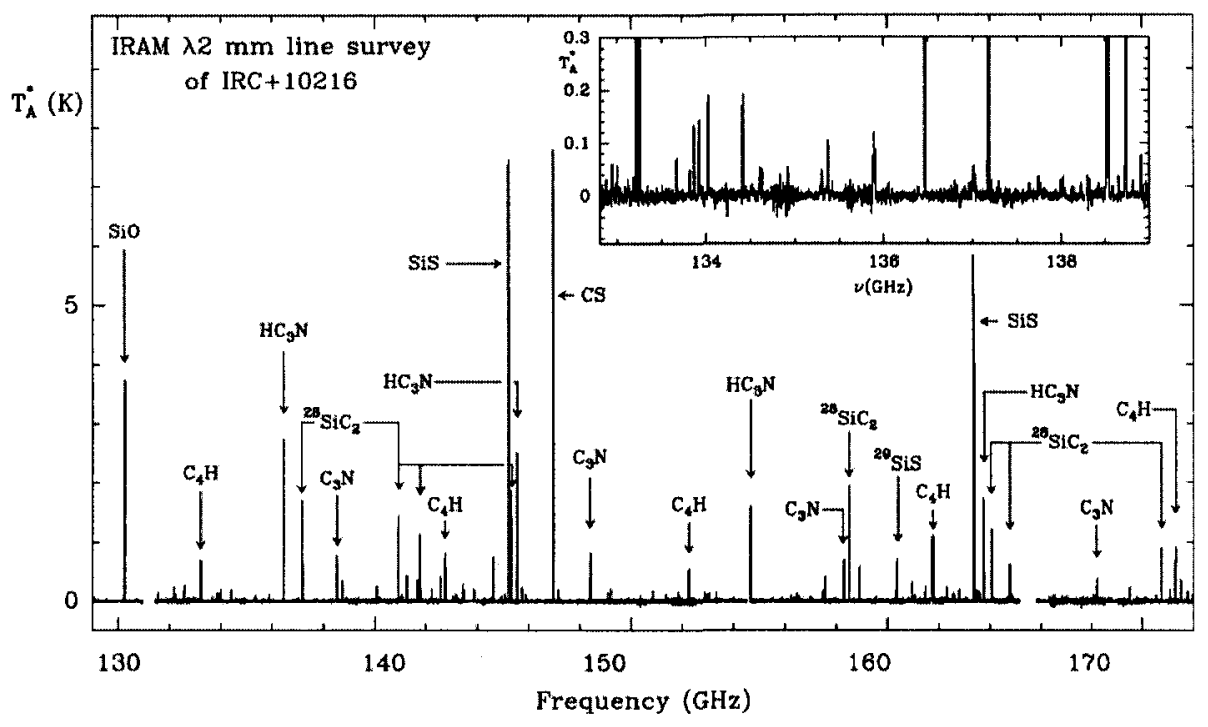

Figure 4. The IRAM $2 \mathrm{~mm}$ spectral survey of IRC+10216, from Cernicharo et al. (2000). The inset shows a closeup of the spectrum between 132.8 and $139 \mathrm{GHz}$. The spectrum is dominated by lines of CS, $\mathrm{SiS}, \mathrm{SiO}, \mathrm{SiC}_{2}, \mathrm{C}_{4} \mathrm{H}, \mathrm{C}_{3} \mathrm{~N}$, and $\mathrm{HC}_{3} \mathrm{~N}$. This figure originally appeared in Astronomy and Astrophysics Supplement Series (Cernicharo, Guélin, $\&$ Kahane 2000, A\&AS, 142, 181) and is reproduced with the kind permission of the Editors.

dust shielding becomes important. As a result, circumstellar molecular clouds are excellent environments for studying photo-chemistry.

While the initial abundances are determined by the equilibrium photospheric chemistry, several important chemical processes take place as the gas flows away from the star:

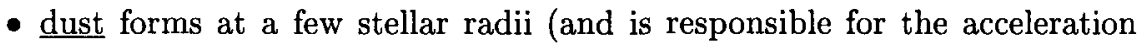
of the wind to its terminal velocity), incorporating a large fraction of the refractory elements;

- complex molecules are photodissociated as outlined above;

- complex chain molecules are built up by ion-molecule reactions in the outer envelope.

These processes are described in detail by Glassgold (1996) and references therein.

Like all molecular clouds, those produced by mass loss have fascinating and complex chemistry. The IRC+10216 cloud, as the brightest circumstellar envelope in the sky, has been the subject of intense study, including spectral line surveys at millimeter and submillimeter wavelengths, which reveal the detailed 
molecular line rotational spectrum of the object. As an example, the $2 \mathrm{~mm}$ molecular line survey by Cernicharo et al. (2000) is shown in Fig. 4.

To date, about 60 molecular and atomic species have been detected in the IRC+10216 envelope (see Kawaguchi et al. 1995; Cernicharo et al. 1996, 2000; Ziurys et al. 2002). These species include:

\section{Atoms: $\mathrm{HI}, \mathrm{CI}$}

The distribution of these species suggests that they are photodissociation products (van der Veen et al. 1995; Le Bertre \& Gérard 2001).

\section{Common molecules: $\mathrm{CO}, \mathrm{CS}, \mathrm{SiO}, \mathrm{CN}, \mathrm{HCN}, \mathrm{SiS}, \mathrm{SiN}$}

These are found in most molecular clouds. The most important factor which determines the circumstellar chemistry is the $\mathrm{C} / \mathrm{O}$ ratio, greater than 1 for IRC+10216. The strong $\mathrm{OH}, \mathrm{SiO}$ and $\mathrm{H}_{2} \mathrm{O}$ maser lines characteristic of circumstellar envelopes with $\mathrm{O} / \mathrm{C}>1$ (see Habing 1996) are absent, as are SO and $\mathrm{SO}_{2}$. Instead, carbon-sulfur molecules like $\mathrm{C}_{2} \mathrm{~S}, \mathrm{C}_{3} \mathrm{~S}$, and $\mathrm{H}_{2} \mathrm{~S}$ are abundant.

The distribution of $\mathrm{SiO}, \mathrm{SiS}$ and $\mathrm{SiN}$ is confined to a few stellar radii, suggesting that these species are incorporated into dust.

\section{Silicon Carbides: $\mathrm{SiC}, \mathrm{SiC}_{2}, \mathrm{SiC}_{3}$}

$\mathrm{SiC}_{2}$ is also seen in many carbon-star photospheres via the Merrill-Sanford bands. These species are of particular interest because of their incorporation into carbonaceous circumstellar grains. The $11.3 \mu \mathrm{m} \mathrm{SiC}$ feature is seen in emission in many carbon star envelopes, and $\mathrm{SiC}$ grains are found as inclusions in some meteorites: thus they are an important tracer of the cycling of the interstellar medium through stars, into mass loss and dust production by evolved stars, and into molecular clouds and star/planetary system formation.

Carbon chains: $\mathrm{C}_{5}, \mathrm{C}_{2} \mathrm{H}, \mathrm{C}_{3} \mathrm{H}_{2}, \mathrm{C}_{4} \mathrm{H}, \mathrm{C}_{5} \mathrm{H}, \mathrm{C}_{6} \mathrm{H}, \mathrm{C}_{7} \mathrm{H}, \mathrm{C}_{8} \mathrm{H}, \mathrm{H}_{2} \mathrm{C}_{4}$

Cyanides and $\mathrm{C}-\mathrm{N}$ chains: $\mathrm{HCCN}, \mathrm{CH}_{3} \mathrm{CN}, \mathrm{C}_{3} \mathrm{~N}, \mathrm{C}_{5} \mathrm{~N}, \mathrm{HC}_{3} \mathrm{~N}, \mathrm{HC}_{5} \mathrm{~N}$, $\mathrm{HC}_{7} \mathrm{~N}, \mathrm{HC}_{9} \mathrm{~N}, \mathrm{HC}_{11} \mathrm{~N}$

\section{Salts, Metals: $\mathrm{KCl}, \mathrm{NaCl}, \mathrm{AlCl}, \mathrm{AlF}, \mathrm{NaCN}, \mathrm{MgNC}$, AlNC}

Most of these are uniquely (so far) found in the IRC+10216 envelope, and they appear to be confined to the inner few stellar radii of the envelope (Cernicharo \& Guélin 1987). These salts, and other molecules such as CO and HCN, are present in many isotopic variants, for example ${ }^{35} \mathrm{Cl}$ and ${ }^{37} \mathrm{Cl}$ are seen in both sodium and aluminum chloride. The determination of isotopic abundances is of great interest for the evolution of the interstellar medium and the effects of the enrichment by stellar winds, and also provide sensitive tracers of the effect of the s-process in the stellar interior. In IRC +10216 , the isotopic abundances of $\mathrm{Mg}, \mathrm{Si}$ and $\mathrm{S}$ are similar to solar, those of $\mathrm{C}$ and $\mathrm{Cl}$ are significantly different, and those of $\mathrm{N}$ and $\mathrm{O}$ are very different from those seen in the Sun. These abundance patterns are characteristic of the s-process for stars of $\leq 2 \mathrm{M}_{\odot}$ (Kahane et al. 2000), in agreement with the inferences on the progenitor mass discussed in Section 2.

Exotica: NP, CP, $\mathrm{H}_{2} \mathrm{O}$ 
Water is exotic in context here: its abundance is expected to be negligible in a carbon star envelope, and water bands are completely absent from the photospheric spectra of carbon stars. Emission from the ground rotational state of $\mathrm{H}_{2} \mathrm{O}$ has been detected in IRC+10216, at an abundance about $10^{-5}$ that of $\mathrm{CO}$, by the SWAS satellite (Melnick et al. 2001). These authors attribute its presence to the vaporization of (relatively) oxygen-rich circumstellar material (comets) left over from formation. IRC+10216 is probably the only mass-losing carbon star bright enough for the detection of water vapor with present technology, so how common this is remains unknown.

Other examples of mixed circumstellar chemistry are known. Something like $10 \mathrm{~J}$-type carbon stars have C-rich atmospheres, $\mathrm{H}_{2} \mathrm{O}$ masers, and both silicate and SiC dust emission features (e.g. Engels 1994; Szczerba et al. 2002). The carbon-rich post-AGB object RAFGL 915 has two circumstellar clouds, one oxygen-rich and one carbon-rich (Waters et al. 1998). The carbon-rich young planetary nebula RAFGL 618 shows infrared lines of $\mathrm{OH}, \mathrm{H}_{2} \mathrm{O}$, and $\mathrm{HI}$ (Herpin \& Cernicharo 2000). All of these observations suggest that circumstellar material of both oxygen-rich and carbon rich composition may be present around some carbon stars, reflecting their chemical evolution on the AGB.

\section{The Envelope Structure and Evolutionary Status of CW Leo}

The envelope of CW Leo can be probed on a wide range of spatial scales, from several arcminutes using $\mathrm{CO}$ and dust tracers to fractions of an arcsecond using Hubble Space Telescope and optical and infrared interferometry. On large spatial scales, the envelope appears to be roughly spherically symmetric (Huggins et al. 1988; Tamura et al. 1988; K. Young \& G. Knapp, unpublished). However, even on these large scales, there are indications of imperfect symmetry and inhomogeneity. Interferometric maps of several molecular species which are emitted from different characteristic radii in the envelope show, superimposed on the regular spherical outflow, significant clumpiness (e.g. Bieging \& Tafalla 1993; Gensheimer et al. 1995; Lucas et al. 1995; Lindqvist et al. 2000). Highsensitivity measurements of the line profiles (e.g. Groenewegen \& Ludwig 1998) show asymmetries which vary with time on scales of a few years. Images of scattered light show multiple shells (Crabtree \& Rogers 1993; Mauron \& Huggins 2000) which suggest episodic mass loss on scales of typically a few hundred years (similar shells are seen in the Egg Nebula, RAFGL 2688, which is at a slightly later stage of evolution than is CW Leo, Sahai et al. 1998). At smaller angular scales, large deviations from symmetry are seen. Images by Kastner \& Weintraub (1994) and Skinner et al. (1998) suggest overall bipolar structure, but more complex structure, with asymmetrically distributed clumps, is suggested by line and continuum interferometry (Haniff \& Buscher 1998; Weigelt et al. 1998; Osterbart et al. 2000; Tuthill et al. 2000; Monnier et al. 2000a; Men'shchikov et al. 2001). Further, Tuthill et al. (2001) show that the structure of the inner envelope is changing on timescales of years: proper motions are seen in individual features.

The lack of spherical symmetry in the inner envelope, and the rapidlychanging mass loss rate evidenced by these changes, has been taken by several authors as evidence that CW Leo is beginning its evolution away from the AGB 
and towards the planetary nebula phase. To be sure, a star which has lost mass at a high rate over the past several tens of thousands of years is clearly reaching the end of its evolution on the AGB, but it is the case that other evolved stars (e.g. the oxygen rich supergiant VY CMa and the carbon star CIT 6) also show clumpiness and rapid changes in the inner envelopes (Monnier et al. 2000a,b). Otherwise, CW Leo has none of the other observational signs of evolution away from the AGB: the development of a very fast molecular wind, or alterations in the circumstellar chemistry (e.g. Herpin \& Cernicharo 2000; Highberger et al. 2001; Herpin et al. 2002). While many more detailed observations are needed to explore the range of variation seen in the structure and kinematics of circumstellar envelopes, the small amount of evidence available at the moment suggests that such asymmetries are an intrinsic part of the evolution of stars on the AGB and will give rise to the bipolar molecular outflows and amazing range of planetary nebula shapes in subsequent evolution (cf. Mastrodemus \& Morris 1999).

Why might such asymmetries happen? The best understanding we have at the moment is that molecular winds around evolved stars are driven by radiation pressure on dust. Since the photospheric temperatures of these huge stars $(<2500 \mathrm{~K})$ are not very different from the condensation temperatures of dust $(\sim 1000 \mathrm{~K})$ small temperature differences across the stellar photosphere will result in aspherical distributions of circumstellar dust. Indeed, the stars themselves have aspherical light distributions (Gilliland \& Dupree 1996; Karovska 1999) which may originate in the very large convection shell structure described by Schwarzschild (1975). The large diameters and cool envelopes of these evolved stars; their rapidly changing luminosity: and the similarities of the convection and pulsation time scales: all of these are likely to cause asphericity in the mass loss. These recent observations of the inner structure of the envelopes of evolved stars suggest that mass loss on the AGB is a much more chaotic process than one might expect, producing variations from star to star in the total amount of mass lost and the chemical composition of the outflow. CW Leo, the closest highly evolved AGB carbon star, is the best object we have to investigate these processes in detail.

Acknowledgments. Thanks to the organizers of this conference for their invitation to attend. This review made extensive use of the SIMBAD data base, operated at CDS, Strasbourg, France, and NASA's Astrophysics Data System.

\section{References}

Becklin, E. E., Frogel, J. A., Hyland, A. R., Kristian, J., \& Neugebauer, G. 1969, ApJ, 158, L133

Bieging, J. H., \& Tafalla, M. 1993, AJ, 105, 576

Chandrasekhar, T., \& Mondal, S. 2001, MNRAS, 322, 356

Cernicharo, J., \& Guélin, M. 1987, A\&A, 183, L10

Cernicharo, J., Guélin, M., \& Kahane, C. 2000, A\&AS, 142, 181

Cernicharo, J., et al. 1996, A\&A, 315, L201

Crabtree, D. R., \& Rogers, C. 1993, in Mass Loss on the AGB and Beyond, ed. H. Schwartz (Garching: ESO), 255 
Crosas, M., \& Menten, K. 1997, ApJ, 483, 913

Dahn, C. C., et al. 2002, AJ, 124, 1170

Engels, D. 1994, A\&A, 285, 492

Foy, R., Chelli, A., Sibille, F., \& Lena, P. 1979, A\&A, 79, L5

Gensheimer, P. D., Likkel, L., \& Snyder, L. E. 1995, ApJ, 439, 445

Gezari, D. Y., Schmitz, M., Pitts, P. S., \& Mead, J. L. 1993, NASA RP-1294

Gilliland, R. L., \& Dupree, A. K. 1996, ApJ, 463, L29

Glassgold, A. E. 1996, ARA\&A, 34, 241

Groenewegen, M. A. T. 1997, A\&A, 317, 503

Groenewegen, M. A. T., \& Ludwig, H.-G. 1998, A\&A, 339, 489

Groenewegen, M. A. T., \& Whitelock, P. A. 1996, MNRAS, 281, 1347

Groenewegen, M. A. T., van der Veen, W. E. C. J., \& Matthews, H. E. 1998 A\&A, 338, 481

Habing, H. J. 1986, A\&AR, 7, 97

Haniff, C. A., \& Buscher, D. F. 1998, A\&A, 334, L5

Herbig, G. H., \& Zappala, R. R. 1970, ApJ, 162, L15

Herpin, J., \& Cernicharo, J. 2000, ApJ, 530, L129

Herpin, F., Goicoechea, J. R., Pardo, J. R., \& Cernicharo, J. 2002, ApJ, in press (astro-ph/0206167)

Highberger, J. L., Savage, C., Bieging, J. H., \& Ziurys, L. M. 2001, ApJ, 562, 790

Huggins, P. J., Olofsson, H., \& Johansson, L. E. B. 1988, ApJ, 332, 1009

Jura, M. 1983, ApJ, 275, 683

Jura, M. 1994, ApJ, 434, 713

Kahane, C., Dufour, E., Busso, M., Gallino, R., Lugaro, M., Forestini, M., \& Straniero, O. 2000, A\&A, 357, 669

Karovska, M. 1999, in IAU Symp. 191, Asymptotic Giant Branch Stars, ed. T. Le Bertre, A. Lèbre, \& C. Waelkens (San Francisco: ASP), 139

Kastner, J. H., \& Weintraub, D. A. 1994, ApJ, 434, 719

Kawaguchi, K., Kasai, Y., Ishikawa, S. I., \& Kaifu, N. 1995, PASJ, 47, 853

Keady, J. J., \& Ridgway, S. T. 1993, ApJ, 406, 199

Knapp, G. R., Bowers, P. F., Young, K., \& Phillips, T. G. 1994, ApJ, 429, L33

Le Bertre, T. 1982, A\&AS, 94, 377

Le Bertre, T., \& Gérard, E. 2001, A\&A, 378, L29

Lindqvist, M., Schöier, F. L., Lucas, R. L., \& Olofsson, H. 2000, A\&A, 361, 1036

Lucas, R., Guélin, M., Kahane, C., Audinos, P., \& Cernicharo, J. 1995, Ap\&SS, 224,293

Mamon, G. A., Glassgold, A. E., \& Huggins, P. J. 1988, ApJ, 328, 797

Mastrodemus, N., \& Morris, M. 1999, ApJ, 523, 357

Mauron, N., \& Huggins, P. J. 2000, A\&A, 359, 707

Melnick, J., Neufeld, D. A., Saavik Ford, K. E., Hollenbach, D. J., \& Ashby, M.

L. 2001, Nature, 412,160 
Men'shchikov, A. B., Balega, Y. Y., Blöcker, T., Osterbart, R., \& Weigelt, G. 2001, A\&A, 368, 497

Monnier, J. D., Danchi, W. C., Hale, D. S., Lipman, E. A., Tuthill, P. G., \& Townes, C. H. 2000a, ApJ, 543, 861

Monnier, J. D., Tuthill, P. G., \& Danchi, W. C. 2000b, ApJ, 545, 957

Morris, M. 1975, ApJ, 197, 603

Neugebauer, G., \& Leighton, R. B. 1969, in Two Micron Sky Survey - A Preliminary Catalog, NASA SP-3047 (Springfield: NTIS), 25

Olofsson, H. 1999, in IAU Symp. 191, Asymptotic Giant Branch Stars, ed. T. Le Bertre, A. Lèbre, \& C. Waelkens (San Francisco: ASP), 3

Olofsson, H., et al. 1996, A\&A, 311, 587

Osterbart, R., Balega, Y. Y., Blöcker, T., Men'shchikov, A. B., \& Weigelt, G. 2000, A\&A, 169

Sahai, R. 1987, ApJ, 318, 809

Sahai, R., et al. 1998, ApJ, 493, 301

Sandell, G. 1994, MNRAS, 271, 75

Schöier, F., \& Olofsson, H. 2001, A\&A, 368, 969

Schwarzschild, M. 1975, ApJ, 195, 137

Skinner, C. J., Meixner, M., \& Bobrowsky, M. 1998, MNRAS, 300, L29

Solomon, P. M., Jefferts, K. B., Penzias, A. A., \& Wilson, R. W. 1971, ApJ, $163, \mathrm{~L} 53$

Szczerba, R., Chen, P. S., Szymczak, M., \& Omont, A. 2002, A\&A, 381, 491

Tamura, M., et al. 1988, ApJ, 326, L17

Truong-Bach, Morris, D., \& Nguyen-Q-Rieu, 1991, A\&A, 249, 435

Tuthill, P. G., Monnier, J. D., Danchi, W. D., \& Lopez, B. 2000, ApJ, 543, 284

van der Veen, W. E. C. J., Omont, A., Habing, H. J., \& Matthews, H. E. 1995, A\&A, 295, 445

Waters, L. B. F. M., et al. 1998, Nature, 391, 868

Weigelt, G., Blöcker, T., Hofmann, K.-H., Osterbart, R., Balega, Y. Y., Fleischer, A. J., \& Winters, J. M. 1999, in IAU Symp. 191, Asymptotic Giant Branch Stars, ed. T. Le Bertre, A. Lèbre, \& C. Waelkens (San Francisco: ASP), 273

Weigelt, G., et al. 1998, A\&A, 333, L51

Winters, J. M., Keady, J. J., Gauger, A., \& Sada, P. V. 2000, A\&A, 359, 651

Young, K., Phillips, T. G., \& Knapp, G. R. 1993, ApJS, 86, 517

Ziurys, L., Savage, C., Highberger, J. L., Apponi, A. J., Guélin, M., \& Cernicharo, J. 2002, ApJ, 564, L45 